\title{
Association of parental characteristics with offspring anthropometric failure, anaemia and mortality in India
}

\author{
Rajesh Kumar Rai (1,2,3,4凶, S. V. Subramanian ${ }^{5,6}$ \& Sebastian Vollmer (1) ${ }^{3,4}$
}

This study used a wide range of information on parental sociodemographic, physical and behavioural characteristics as well as on the presence of non-communicable diseases among parents and examined the association of these attributes with anthropometric failure, anaemia and mortality of their children aged 0-59 months. Findings revealed that children of fathers aged 30-39 years were less likely to experience anthropometric failure and anaemia; however, survival of children of fathers below 18 years at marriage could be threatened. Parental education had protective association with children's anthropometric failure, anaemia and under-five mortality. With increasing maternal height, children had lower odds of anthropometric failure and under-five mortality. Tobacco use by mothers was associated with increase in under-five mortality, and children with diabetic fathers had higher odds of underfive mortality.

\footnotetext{
${ }^{1}$ Society for Health and Demographic Surveillance, Suri, West Bengal, India. ${ }^{2}$ Department of Global Health and Population, Harvard T H Chan School of Public Health, Boston, MA, USA. ${ }^{3}$ Department of Economics, University of Goettingen, Göttingen, Germany. ${ }^{4}$ Centre for Modern Indian Studies, University of Goettingen, Göttingen, Germany. ${ }^{5}$ Harvard Center for Population and Development Studies, Cambridge, MA, USA. ${ }^{6}$ Department of Social and Behavioral Sciences, Harvard T H Chan School of Public Health, Boston, MA, USA. 凶email: rajesh.iips28@gmail.com
} 


\section{Introduction}

volutionary biologists have extensively studied the importance of parental characteristics in shaping their offspring's health and the pathways through which this relationship operates (Wells, 2017). The pathways start with children's intrauterine exposure, maternal exposure during pregnancy, followed by parents' choices about the amount and quality of healthcare their children receive, the food they eat, the amount of emotional support they are provided and the quality of the environment in which they live (Cutler et al., 2008; Bauman et al., 2006; Case and Paxson, 2002). These choices are often conditioned by parents' material resources, knowledge of health practices and programmes, their own health behaviour and the characteristics of the communities they live in (Case and Paxson, 2002; Case et al., 2002).

As a first step of this study, a literature search on PubMed/ MEDLINE bibliographic database was conducted using appropriate Medical Subject Headings (MeSH) terms to learn about the existing studies on the relationship between parental characteristics and offspring's health, with a focus on India. While searching relevant literature, emphasis was laid on studies that analysed parental sociodemographic and behavioural characteristics, and parental health status and their effects on offspring health. Of sociodemographic characteristics, the literature search suggested that child marriage of girls (married before age 18) in India was associated with increased risk of offspring morbidity and mortality (Kumar et al., 2021a; Fall et al., 2015; Finlay et al., 2011; Raj et al., 2010). When it comes to child marriage of boys, the literature is rather scarce. Child marriage of boys is likely to constrain their access to education and opportunities for career advancement (Gastón et al., 2019), which could potentially limit their earnings and their access to healthcare and healthcare information. A study analysed Demographic and Health Survey (DHS) data from India and concluded that children (aged 12-59 months) of younger parents (aged 15-24 years at the time of survey) had elevated risk of anaemia, stunting and incomplete immunisation (Puri et al., 2020). A longitudinal study for over 15 years (Perez-Alvarez and Favara, 2019) on a total of 12,000 children from Ethiopia, India (from the states of Andhra Pradesh and Telangana), Peru and Vietnam revealed that children born to early mothers are shorter for their age and perform poorer in mathematics tests. A research collaboration of five birth cohorts from low- and middle-income countries, such as Brazil, Guatemala, India, Philippines and South Africa, found that maternal age of $\leq 19$ years was associated with increased risk of low birthweight, preterm birth and stunting among children of 2 years of age (Fall et al., 2015). Using cross-sectional data from 118 DHSs conducted in 55 countries (between 1990 and 2008), a study found that mothers below the age of 27-29 years had elevated risks for infant mortality in first-born children and stunting, diarrhoea and anaemia in all children (Finlay et al., 2011).

Recent studies have demonstrated that children of uneducated parents are more likely to experience poor health outcomes as compared to children with educated parents in India (Kumar et al., 2021b; Puri et al., 2020; Srivastava and Upadhyay, 2022). Studies have also found that maternal education has a protective association with double burden of malnutrition (Patel et al., 2020) and triple burden of malnutrition among their children (Kumar et al., 2021a; Kumar et al., 2021b). A global systematic review and meta-analysis (Balaj et al., 2021) found that not only illiteracy but also lower maternal and paternal education are risk factors for child mortality, whereas an analysis of 80 DHS datasets from 62 countries revealed that higher maternal and paternal educational levels were associated with lower childhood anthropometric failures (Vollmer et al., 2017). While research about the stronger effects of education of either parent remains inconclusive, researchers have urged to count on both maternal and paternal education for improving the health of the offspring (Balaj et al., 2021; Vollmer et al., 2017). Like parental education, parental employment is also an indicator of financial security, which is often conditioned by education of a certain level. A recent study documented that if both parents are employed, their children were less likely to have incomplete immunisation; however, no association with childhood anaemia and stunting was found (Puri et al., 2020). Children with mothers employed in professional jobs had better health status as compared to children whose mothers were employed in agricultural and manual (skilled and unskilled) jobs (Shajan and Sumalatha, 2020).

The association of parental phenotypic traits such as height, weight and body mass index (BMI) with children's health has also been widely studied. Using DHS data from India, a study documented that increased height of both parents was associated with decreased odds of childhood stunting (Gupta et al., 2021). In the wake of the debate around intrauterine influences on child nutrition, a study used nationally representative data from India and found that higher values of maternal and paternal BMIs were associated with higher values of offspring BMI (Corsi et al., 2015). However, while comparing the effect sizes of maternal and paternal BMIs, no consistent differences were found in the strength of these parental associations with the BMI of the offspring. This finding resonates with an earlier study, which found that a one unit increase of maternal BMI was associated with lower relative risk of childhood stunting, wasting and underweight (Subramanian et al., 2010). Another study used data from 109 DHSs of 54 countries and showed that a $1 \mathrm{~cm}$ increase in maternal height was associated with decreased risk of offspring mortality, underweight and stunting in infancy and childhood (Özaltin et al., 2010). In line with these studies, Subramanian et al. (2009) documented that an increased maternal height of $1 \mathrm{~cm}$ was inversely associated with child mortality, stunting, wasting, underweight and childhood anaemia in India.

Of behavioural characteristics, parental use of tobacco could be detrimental to the offspring's health (Zhuge et al., 2020; Caleyachetty et al., 2014). Exposure of unborn children to maternal smoking during pregnancy or second-hand smoke is linked to birth defects, stillbirths, preterm births and infant deaths (Nicoletti et al., 2014; Leonardi-Bee et al., 2011). Newborns and young children who are exposed to tobacco smoke in their immediate environment are at increased risk of asthma, respiratory infections and meningococcal disease, leading to increased mortality (Faber et al., 2017). In addition, tobacco consumption among adults comes with significant cost to families, contributing to hunger and food insecurity for both children and adults in both higher-income and lower-income countries (World Health Organization, 2021). Many households are impoverished by the cost of smoking, diverting spending away from basic household expenses with an increased risks of catastrophic health expenditure (Semba et al., 2007). A cross-sectional study utilizing data from eight South Asian and South East Asian countries documented that child death was associated with smoking by both parents, parental use of smokeless tobacco and use of any tobacco (Bhatta and Glantz, 2019). Like tobacco use, alcohol misuse or problematic drinking behaviour of parents is associated with poor child health, cognitive development, behavioural problems in younger children and a range of problematic behaviours in adolescence such as absence from school, substance abuse and teenage pregnancy (Huq et al., 2021; Jose and Cherayi, 2020). Consumption of alcohol prior to and during pregnancy could lead to an increased risk of adverse birth outcomes such as low birthweight and preterm birth (Nykjaer et al., 2014). A study conducted among young Swedish males revealed that paternal 
alcohol consumption was associated with increased risk of offspring mortality (Landberg et al., 2018).

The presence of non-communicable diseases (NCDs) such as diabetes mellitus and hypertension (elevated blood pressure) among parents could also lead to poor offspring health. While diabetes and hypertension could affect both rich and poor, parental NCDs could be an indication of inaccessibility to good healthcare practices or insufficient healthcare information. A study conducted in the United States concluded that maternal diabetes prior to or during pregnancy was associated with poorer glycaemic control and $\beta$-cell function among their children (Chernausek et al., 2016). Using 8301 mother-offspring pairs, a study concluded that maternal diabetes is associated with high blood pressure late in childhood, demonstrating the role of children's body mass in the pathway of this association (Miranda et al., 2019). Like diabetes, parental hypertension is associated with poor child health outcomes (Miliku et al., 2016), and pathways through which it affects child health could be genetic as well (Uehara et al., 1998).

From this overview, it is evident that the majority of published studies had measured a specific indicator of child's health and its association with a limited range of parental characteristics, which limits our understanding of this issue. For example, one drawback of earlier studies in India is that none of them examined the presence of NCDs among parents and its relationship with offspring health. Against this evidence gap, using a nationally representative cross-sectional dataset from India, a comprehensive set of information on parental sociodemographic, physical and behavioural characteristics, and on the presence of NCDs among parents was employed to examine whether these parental attributes were associated with anthropometric failure, anaemia and mortality of their children aged 0-59 months. Findings from this study could be useful in devising a strategy for mitigating the burden of anthropometric failure, anaemia and mortality among children aged under 5 in India.

\section{Methods}

Study design and data. This study used data from the nationally representative fourth wave of the standard Demographic and Health Survey (DHS) of India, commonly known as the 2015-2016 National Family Health Survey or NFHS-4 (International Institute for Population Sciences and ICF, 2017). Health statistics from NFHS-4 are used extensively to guide district, state and national public health policy in India. NFHS-4 is crosssectional and covers 640 districts from 37 states or union territories. India's 2011 Census sampling frame was used to select primary sampling units (PSUs) in rural and urban areas, and NFHS-4 adopted a stratified two-stage sampling design. From the predefined sampling frame, PSUs were identified as villages in rural areas and Census Enumeration Blocks (CEBs) in urban areas. Within each rural stratum, villages were selected from the sampling frame with probability proportional to size (PPS). In each stratum, six approximately equal substrata were created by crossing three substrata, each created based on the number of households in each village, with two substrata, each created based on the percentage of the population belonging to Scheduled Castes (SCs) and Scheduled Tribes (STs). In urban areas, CEBs were sorted according to the percentage of the SC/ST population in each CEB, and sample CEBs were selected with the PPS sampling method. NFHS-4 had a household response rate of $97.6 \%$. The NFHS-4 sampled 699,686 women aged 15-49 years and 112,122 men aged 15-54 years residing in 601,509 households for the interview. Further details about the sampling procedure of NFHS- 4 can be reviewed from its published report (International Institute for Population Sciences and ICF, 2017).
A dataset with parent-child dyads was prepared by merging three separate datasets with information on children, men and all household members. The purpose of merging these datasets was to have a single dataset with information of parents (both father and mother) linked to their offspring. Using appropriate merging identifiers, the household member dataset was merged with men's dataset at the first stage; and at the second stage, the merged dataset from the first stage was re-merged with the children's file. The children's file had information for 259,627 children aged 0-59 months (247,743 living children and 11,884 deceased children). Upon merging required datasets (children, men and all household members), 33,047 children aged 0-59 months were identified with information on both parents. Among these children aged 0-59 months, 28,952 children were eligible for calculating anthropometric failure, whereas 27,265 children aged 6-59 months were measured for childhood anaemia. As a final sample included in the analysis, 25,429 under-five children were found eligible for analysing anthropometric failure, and 28,693 for under-five mortality, whereas 24,022 children aged 6-59 months were eligible to be included for analysing anaemia. Derivation of sample size is presented schematically as an online supplement (Fig. S1). Samples included in the analysis were compared with the samples excluded from the analysis for three outcome events (anthropometric failure, anaemia and under-five mortality) by selected socioeconomic characteristics of children to assess the possible extent of sample selection bias in this study.

Outcome variables: anthropometric failure, anaemia and under-five mortality. Outcome events were chosen in two stages. At stage I, the association with parental characteristics was analysed for three outcome events-any anthropometric failure, anaemia and under-five mortality, and they were labelled as primary outcome measures. At stage II, as complementary outcome measures, anthropometric failure was categorised into underweight, stunting, wasting, severe underweight, severe stunting and severe wasting; anaemia was further analysed for mild-moderate anaemia and severe anaemia; and in addition to under-five mortality, neonatal mortality and postneonatal mortality were also investigated. All complementary outcome measures were tested for their association with parental characteristics; findings are available in the online supplement.

While any anthropometric failure and mortality were measured among children aged 0-59 months, the measure of anaemia was restricted to children aged 6-59 months in NFHS-4. Children identified as being either stunted, wasted or underweight were defined as having any anthropometric failure. Guided by the methodology developed by the World Health Organization (WHO Multicentre Growth Reference Study Group, 2006), stunting was defined as $<-2$ standard deviations from median height for age of the reference population; wasting was defined as $<-2$ standard deviations from median weight for height of the reference population; and underweight was defined as $<-2$ standard deviations from median weight for age of the reference population. Severe stunting, severe wasting and severe underweight were defined as $<-3$ standard deviations from median height for age, weight for height and weight for age of the reference population, respectively (WHO Multicentre Growth Reference Study Group, 2006). For child anthropometry, in NFHS-4, the 'SECA 874 U digital scale' was used for weighing, the 'SECA 213 Stadiometer' was used for measuring height and the 'SECA 417 Infantometer' was used for measuring the length of children under 2 years or less than $85 \mathrm{~cm}$ (International Institute for Population Sciences, 2014). Children aged 6-59 months diagnosed with haemoglobin level (recorded in grams/decilitre or $\mathrm{g} / \mathrm{dl}$ ) of $<11.0 \mathrm{~g} / \mathrm{dl}$ were defined as anaemic, 
whereas children diagnosed with haemoglobin level of 7.0-10.9 g/dl and haemoglobin level of $<7.0 \mathrm{~g} / \mathrm{dl}$ were defined as mild to moderate or severe anaemia, respectively (World Health Organization, 2011). NFHS-4 provides Hb estimates of children adjusted for the altitude of their residence (ICF, 2018; Sullivan et al., 2008). Using HemoCue $\mathrm{Hb} 201+$ machines, NFHS-4 measured $\mathrm{Hb}$ levels from a capillary blood sample of children aged 6-59 months. Death of a child during the first 28 days of life was defined as neonatal mortality, deaths between 28 and 364 days of life were considered as postneonatal mortality, and death of children between birth and their fifth birthday were defined as under-five mortality. Information on mortality was based on recall of age at death from mothers for all children ever born. Detailed protocols about obtaining data for child anthropometry and child biomarkers were presented in the clinical anthropometric biochemical manual of NFHS-4 (International Institute for Population Sciences, 2014).

Predictors: parental characteristics and covariables. Parental characteristics were divided into four categories, namely sociodemographic, physical and behavioural characteristics, and presence of selected NCDs. Sociodemographic characteristics included age group (15-29, 30-39 and $\geq 40$ years), age at marriage $(<18,18-20,21-25$ and $\geq 26$ years), education (no or incomplete primary, primary or incomplete secondary and secondary or higher), years of schooling and employment (unemployed, nonmanual, agricultural and manual/others). Physical characteristics included person's height $(<145,145-149.9,150-154.9,155-159.9$ and $\geq 160 \mathrm{~cm}$ ) and body mass index (BMI in kilogram per metre squared: $\quad<17.00, \quad 17.00-18.49, \quad 18.50-23.49, \quad 23.50-24.99$, $25.00-29.99$ and $\geq 30.00$ ). Behavioural characteristics of parents included current use of tobacco (non-user and user) and alcohol (non-user and user), whereas presence of NCDs among parents included their clinically diagnosed hypertension (non-hypertensive and hypertensive) and diabetes status (non-diabetic and diabetic).

In addition, maternal age at birth $(<17,17-19,20-24,25-29$ and 30-49 years), child's age (0, 1, 2, 3 and 4 years), child's sex (male and female), child's birth order (first, second, third, fourth and fifth or higher), area of residence (urban and rural), religion (Hinduism, Islam, Christianity and Sikhism or others), social group (SCs, STs, Other Backward Classes and Others), wealth index and state of residence (non-high-focus group states and high-focus group states) were included as potential predictors. Inclusion and computation of the variables was guided by a range of existing literature focused on assessing the effect of parental characteristics on health of their children in India and other lowand middle-income countries (Kumar et al., 2021a; Kumar et al., 2021b; Puri et al., 2020; Patel et al., 2020; Vollmer et al., 2017; Rai et al., 2017; Sinha et al., 2016; Corsi et al., 2015; Subramanian et al., 2010; Özaltin et al., 2010; Subramanian et al., 2009). More about the construction and definition of variables in NFHS-4 can be obtained from the DHS recode manual VII (ICF, 2018).

As per the Constitution of India, social groups such as the SCs, STs and the so-called Other Backward Classes are historically, socially and economically disadvantaged populations; the 'Others' category represents the population that has historically been more privileged (Rai et al., 2022). Wealth index was calculated by NFHS-4 based on household assets and durables (Vyas and Kumaranayake, 2006). Owing to both high fertility and highmortality indicators, nine states are regarded as high-focus group states in need of special attention; these are Bihar, Chhattisgarh, Jharkhand, Madhya Pradesh, Odisha, Rajasthan, Uttarakhand, Uttar Pradesh and Assam (Rai et al., 2022). BMI was calculated by dividing the weight in kilograms by the height in metres squared $\left(\mathrm{kg} / \mathrm{m}^{2}\right)$. To measure the status of diabetes, random blood glucose was measured. Individuals with a blood glucose level of $\geq 141$ milligrams per decilitre or those on medication for diabetes were defined as diabetic (Geldsetzer et al., 2018). Individuals were defined as hypertensive if their systolic blood pressure level reading was $\geq 140$ millimetres of mercury ( $\mathrm{mmHg}$ ), or diastolic blood pressure reading was $\geq 90 \mathrm{mmHg}$, or if they were on medication to control hypertension (Geldsetzer et al., 2018). For measuring adult BMI, a SECA 874 U digital scale was used to measure weight, whereas the SECA 213 Stadiometer was used for measuring the height of adults. The standardized protocol for measuring capillary blood sample for glucose level, and measurement of blood pressure were presented in the clinical anthropometric biochemical manual of NFHS-4 (International Institute for Population Sciences, 2014).

Statistical analysis. The sample included in the analysis was compared with the sample excluded from the analysis for all three outcome events-anthropometric failure, anaemia and under-five mortality, for select background characteristics of children (sex of children, education of mother, age at birth of mothers, sex of the household head, social group and wealth index) using a chisquared test of independence to assess the presence of sample selection bias. Variance inflation factor (VIF) was estimated to assess the presence of multicollinearity among predictor variables for all primary and complementary outcome measures, and a VIF of more than 5 against a predictor variable was considered as multicollinear (Chatterjee and Hadi, 2013). Weighted multivariable logistic regression was used to understand the association between parental characteristics and all primary (any anthropometric failure, any anaemia and under-five mortality) and complementary outcome measures (underweight, stunting, wasting, severe underweight, severe stunting, severe wasting, mildmoderate anaemia, severe anaemia, neonatal mortality and postneonatal mortality). For each outcome event, three separate multivariable regression analyses were conducted. Model I was adjusted for paternal characteristics, Model II included maternal characteristics and Model III was adjusted for parental (both father and mother) characteristics. All regression models were adjusted for paternal or/and maternal characteristics and potential socioeconomic predictors-maternal age at birth, child age (for modelling anthropometric failure and anaemia only), sex, birth order, area of residence, religion, social group, wealth index and states of residence. Appropriate sample weights, available with the NFHS-4 dataset, were used. Analysis was performed using the 'svy' suite available with statistical software Stata version 14 (StataCorp, 2015).

\section{Results}

Guided by the existing literature, the socioeconomic characteristics for assessing sample selection bias were sex of the child, education of mother, age at birth of mother, sex of the household head, social group and wealth index (Table S1), and the sample selected for the analysis was not different $(p>0.05)$ from the sample excluded due to ineligibility, indicating the absence of sample selection bias. Table 1 represents the sample distribution of the sample selected for anthropometric failure, anaemia and under-five mortality by characteristics of children. Of the total sample, over $50 \%$ of fathers were aged 30-39 years, whereas over $70 \%$ mothers were from the 15-29 age group. Most fathers got married between ages 21 and 25, in contrast to mothers who typically got married under 18 years of age. Over $20 \%$ of fathers had either no education or completed primary education, whereas the proportion of mothers in the same group was more than $30 \%$. Around $6 \%$ of fathers were unemployed, while over $75 \%$ mothers 
Table 1 Sample distribution of children selected to measure their anthropometric failure (aged 0-59 months), anaemia status (aged 6-59 months) and mortality (aged 0-59 months), by parental characteristics and other covariates.

Anthropometric failure

n (weighted \%, or mean)

Parental current age (in years)

Father

15-29

$30-39$

$\geq 40$

Mother

15-29

30-39

$\geq 40$

Parental age at marriage (years)

Father

$<18$

$18-20$

21-25

$\geq 26$

Mother

$<18$

$18-20$

21-25

$\geq 26$

Parental education

Father

No or incomplete primary

Primary or incomplete secondary

Secondary or higher

Mother

No or incomplete primary

Primary or incomplete secondary

Secondary or higher

Parental years of schooling (mean, SE)

Father

Mother

Parental employment

Father

Unemployed

Non-manual

Agricultural

Manual /others

Mother

Unemployed

Non-manual

Agricultural

Manual /others

Parental height (in $\mathrm{cm}$ )

Father

$<145$

$145-149.9$

150-154.9

155-159.9

$\geq 160$

Mother

$<145$

$145-149.9$

150-154.9

155-159.9

$\geq 160$

Parental height (mean, SE)

Father

Mother

Parental BMI

Father

$<17.00$

$17.00-18.49$

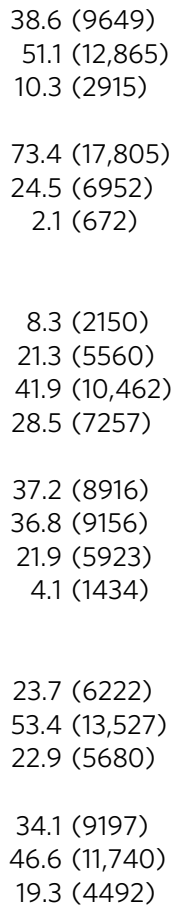

38.6 (9649)

$51.1(12,865)$

10.3 (2915)

$73.4(17,805)$

$24.5(6952)$

$2.1(672)$

8.3 (2150)

$21.3(5560)$

$41.9(10,462)$

28.5 (7257)

37.2 (8916)

36.8 (9156)

21.9 (5923)

4.1 (1434)

23.7 (6222)

$53.4(13,527)$

22.9 (5680)

34.1 (9197)

$46.6(11,740)$

19.3 (4492)

$7.7(0.0)$

$6.6(0.0)$

6.5 (1542)

25.5 (6128)

33.5 (9236)

34.6 (8523)

$76.3(19,004)$

$4.6(1300)$

$13.3(3658)$

5.9 (1467)

$0.6(106)$

$1.7(492)$

7.4 (1961)

$18.9(5022)$

$71.4(17,848)$

11.9 (2983)

$26.0(6676)$

34.2 (8674)

20.1 (5179)

7.8 (1917)

$163.6(0.4)$

$151.7(0.4)$

$5.3(1230)$

11.5 (2877)
Anaemia

n (weighted \%, or mean)

Mortality

n (weighted \%, or mean)
$38.7(10,930)$

$50.8(14,416)$

10.5 (3347)

$73.1(20,075)$

$24.6(7823)$

$2.3(795)$

$8.3(2453)$

$21.4(6316)$

$42.1(11,822)$

28.2 (8102)

$37.3(10,135)$

$36.6(10,324)$

$22.0(6652)$

4.1 (1582)

$24.0(5937)$

$53.5(12,781)$

22.5 (5304)

34.4 (8798)

$46.4(11,028)$

19.2 (4196)

$7.7(0.0)$

$6.5(0.0)$

6.5 (1460)

$25.2(5754)$

$33.9(8766)$

$34.4(8042)$

$75.5(17,791)$

4.7 (1245)

13.7 (3549)

$6.1(1437)$

$0.6(106)$

$1.8(479)$

7.6 (1938)

19.2 (4747)

$70.8(16,752)$

$12.0(2845)$

26.0 (6309)

34.2 (8170)

19.9 (4861)

7.9 (1837)

$163.5(0.5)$

$151.7(0.4)$

$5.2(1163)$

11.2 (2660)
$24.4(7202)$

$52.9(15,186)$

22.7 (6305)

$34.7(10,546)$

$46.1(13,169)$

19.2 (4978)

$7.7(0.0)$

$6.5(0.0)$

6.6 (1771)

$25.3(6845)$

$33.8(10,485)$

34.4 (9592)

$76.1(21,410)$

4.7 (1472)

13.5 (4170)

5.7 (1641)

$0.6(135)$

$1.8(579)$

7.6 (2276)

$19.0(5694)$

$70.9(20,009)$

$12.3(3466)$

26.3 (7585)

33.9 (9717)

19.8 (5767)

7.7 (2158)

$163.5(0.4)$

$151.7(0.4)$

5.3 (1397)

11.5 (3230) 
Table 1 (continued)

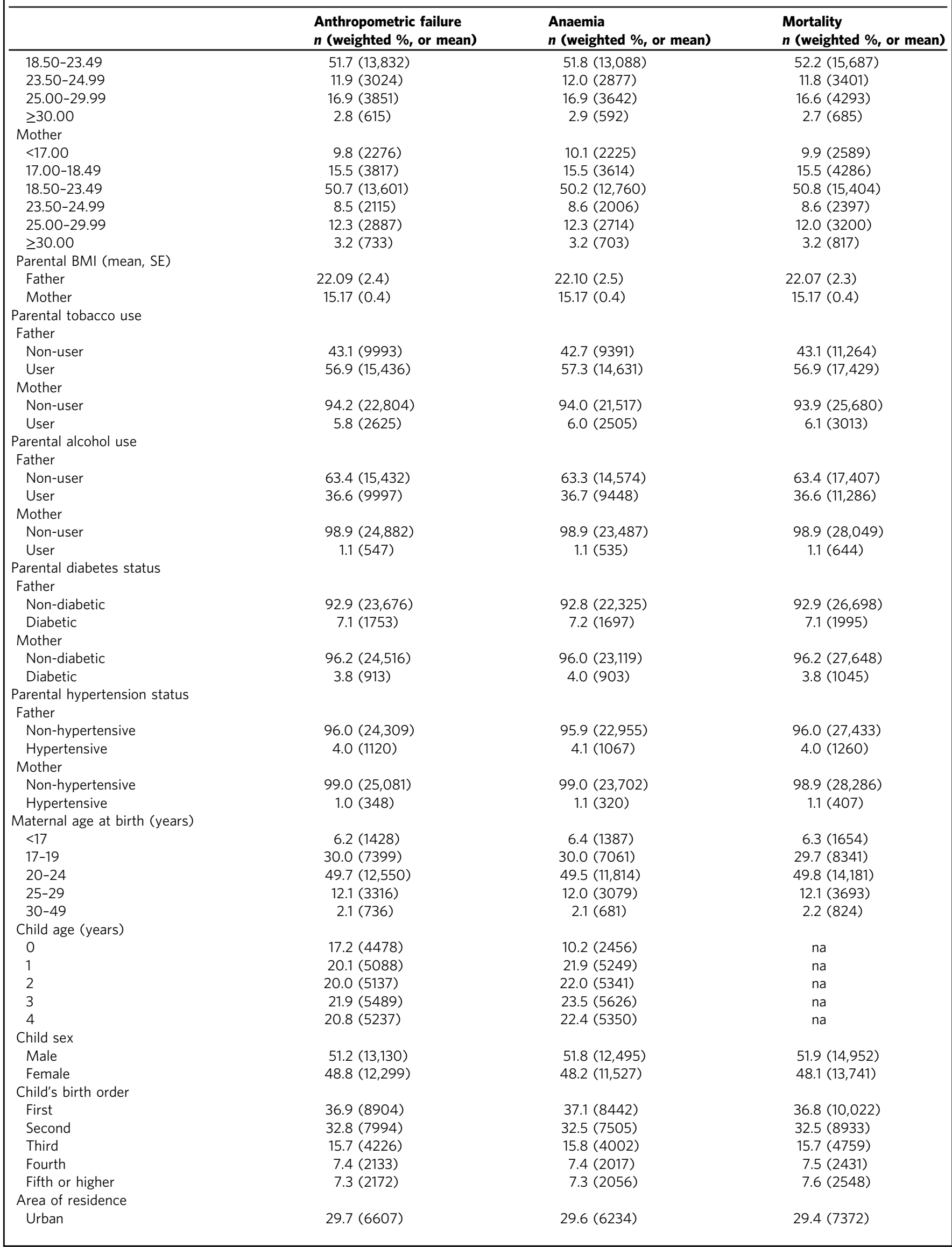


Table 1 (continued)

\begin{tabular}{|c|c|c|c|}
\hline & $\begin{array}{l}\text { Anthropometric failure } \\
n \text { (weighted \%, or mean) }\end{array}$ & $\begin{array}{l}\text { Anaemia } \\
n \text { (weighted \%, or mean) }\end{array}$ & $\begin{array}{l}\text { Mortality } \\
n \text { (weighted \%, or mean) }\end{array}$ \\
\hline Rural & $70.3(18,822)$ & $70.4(17,788)$ & $70.6(21,321)$ \\
\hline \multicolumn{4}{|l|}{ Religion } \\
\hline Islam & $13.3(3280)$ & $13.2(3085)$ & $13.1(3678)$ \\
\hline Christian & $2.2(2005)$ & $2.3(1882)$ & $2.3(2285)$ \\
\hline Sikhs & $1.4(456)$ & $1.4(413)$ & $1.4(498)$ \\
\hline Others & $20.0(4604)$ & $20.2(4362)$ & $19.8(5087)$ \\
\hline Scheduled castes & $21.9(5041)$ & $21.8(4786)$ & $21.8(5705)$ \\
\hline Scheduled tribes & $11.4(5462)$ & $11.4(5102)$ & 11.7 (6259) \\
\hline Other backward classes & $46.7(10,322)$ & 46.7 (9772) & $46.8(11,642)$ \\
\hline \multicolumn{4}{|l|}{ Wealth index } \\
\hline Poorest & $22.8(6207)$ & $22.9(5908)$ & $23.1(7130)$ \\
\hline Non-high-focus group states & $48.3(10,738)$ & $48.9(10,230)$ & $48.4(12,124)$ \\
\hline High-focus group states & $51.7(14,691)$ & $51.1(13,792)$ & $51.6(16,569)$ \\
\hline Total & 25,429 & 24,022 & 28,693 \\
\hline
\end{tabular}

were unemployed. Most parents had a BMI of $18.50-23.49 \mathrm{~kg} / \mathrm{m}^{2}$. Use of tobacco and alcohol among fathers was higher than among mothers, as was the proportion of having diabetes and hypertension.

Tables 2-4 represent associations between parental characteristics and any anthropometric failure, any anaemia and under-five mortality measured as odds ratio (OR) with a $95 \%$ confidence interval (CI). VIFs for all predictor variables were $<5$, indicating a low probability of multicollinearity (results not shown separately). Interpretation and discussion of findings focus on models, which included both parents' characteristics (that is Model III), unless mentioned otherwise. This strategy of interpretation followed the consensus that both parents' characteristics generally have a joint effect on their offspring's health. Findings revealed that as compared to fathers aged 15-29 years, children of fathers aged 30-39 years were less likely to have any anthropometric failure or any anaemia $(\mathrm{OR}=0.87, \mathrm{CI}=0.77-0.97$ and $\mathrm{OR}=0.88, \mathrm{CI}=0.79-0.98$, respectively), and a similar association was observed for Model I, which adjusted only paternal characteristics. Compared to fathers who got married before 18 years of age, children born to fathers who were married between ages 18 and 25 years had lower odds of under-five mortality. Parental education appeared to be a protective factor for all three primary outcomes-any anthropometric failure, any anaemia and under-five mortality. This association was also found in Models I and II, which independently controlled for paternal and maternal characteristics, respectively. With increasing maternal height, children had lower odds of any anthropometric failure and were less likely to experience under-five mortality. This association was also found after controlling for maternal characteristics only (Model II). Increased BMI of fathers $\left(18.50\right.$ through $\geq 30 \mathrm{~kg} / \mathrm{m}^{2}$ ) and mothers was associated with lower anthropometric failure, whereas mothers' BMI (18.50 through $\left.29.99 \mathrm{~kg} / \mathrm{m}^{2}\right)$ was associated with lower odds of anaemia. Use of tobacco by mothers was associated with increased under-five mortality $(\mathrm{OR}=1.50$, $\mathrm{CI}=1.17-1.91$ ), as evident from Model II (adjusted for maternal characteristics) and Model III (adjusted for parental characteristics). Children of fathers with diabetes had higher odds of under-five mortality $(\mathrm{OR}=1.36, \mathrm{CI}=1.01-1.82)$, which was also found in Model I. Children born to mothers with hypertension had lower odds $(\mathrm{OR}=0.69, \mathrm{CI}=0.51-0.94)$ of any anthropometric failure (Model III), which was also found in Model II. The analyses of complementary outcome measures are presented in online supplements-underweight (Table S2), stunting (Table S3), wasting (Table S4), severe underweight (Table S5), severe stunting (Table S6), severe wasting (Table S7), mild-moderate anaemia (Table S8), severe anaemia (Table S9), neonatal mortality (Table S10) and postneonatal mortality (Table S11). To reiterate, the complementary analyses have been supplied to understand the role of parental characteristics on various stages of any anthropometric failure, any anaemia and under-five mortality of their children.

\section{Discussion}

Parental characteristics and their relationship with offspring health have been studied widely, mostly by evolutionary biologists. Public health researchers have attempted to explore if the evolutionary aspect of parent-child relationship could help mitigate some of the most daunting challenges in child health (Wells et al., 2017). Existing studies on the role of parental characteristics on offspring health in India have tested a limited range of information on parental attributes and often tested limited indicators of child health. To address this knowledge gap, this study used a nationally representative cross-sectional NFHS4 dataset to expand and understand the role of additional parental characteristics on the health of their children aged 0-59 months. Three primary outcome measures were analysed-any anthropometric failure, anaemia and under-five mortality; the discussion of this article has focused on these primary outcome measures, while complementary analyses were supplied for further understanding and review of the issue. 


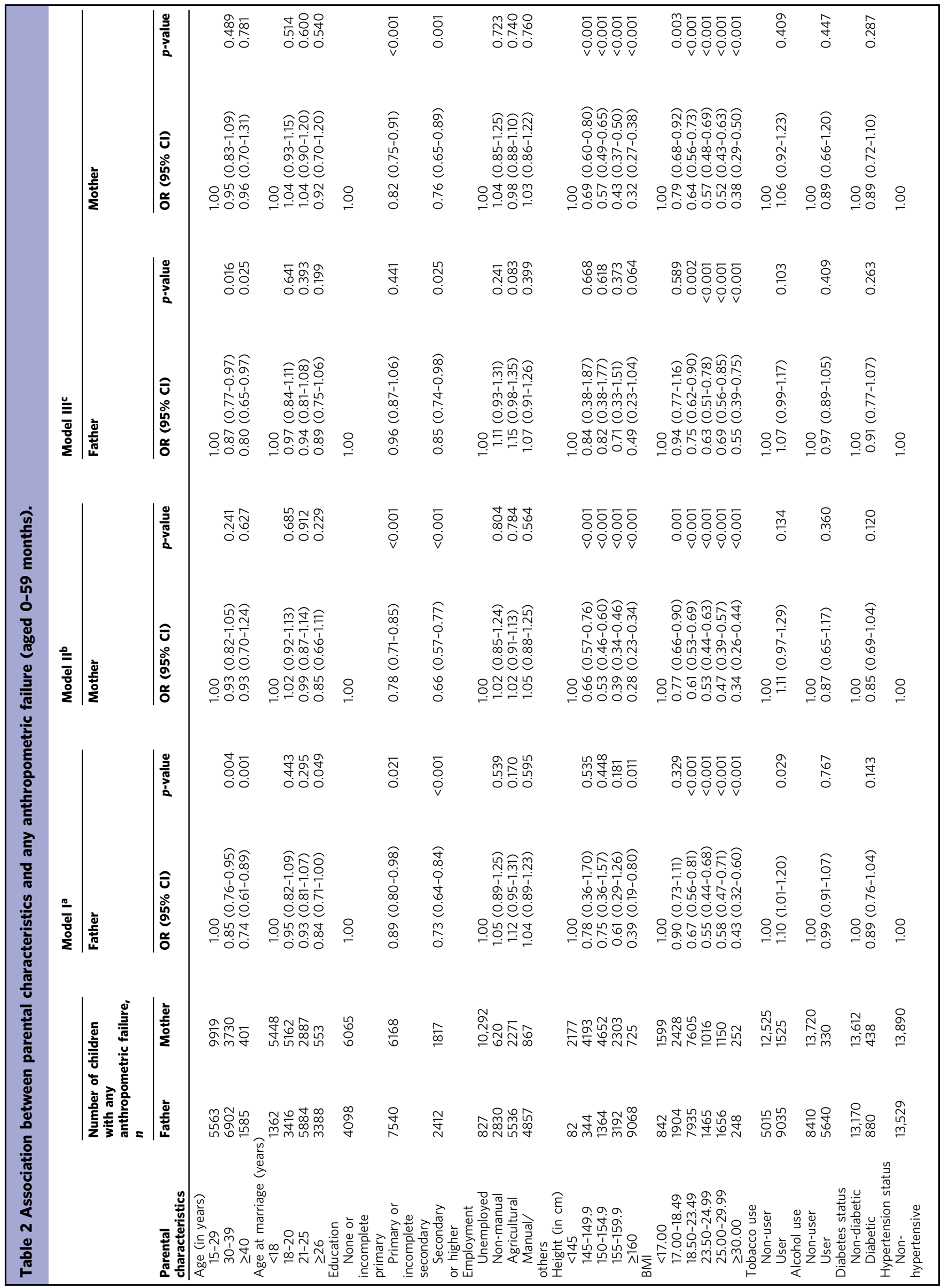




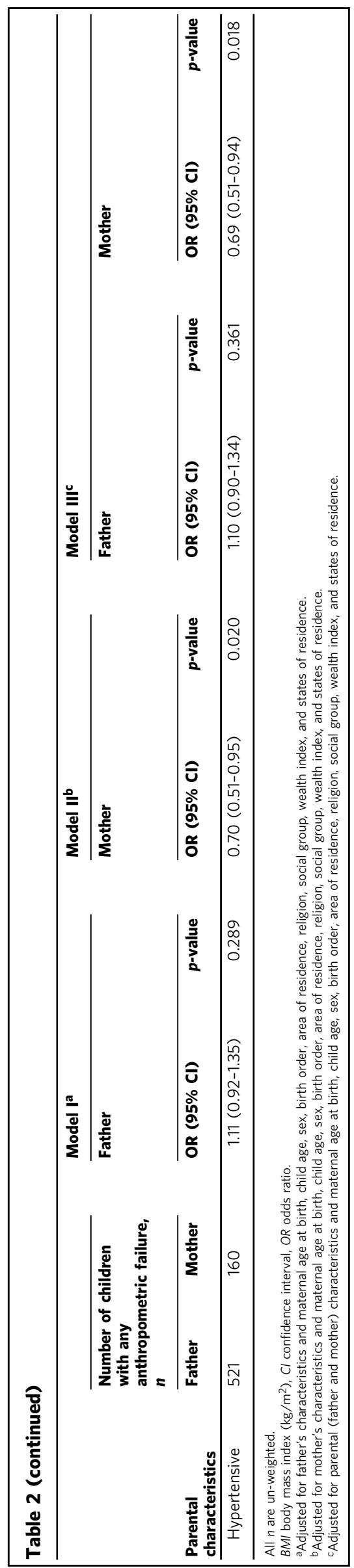

Findings of this study should be interpreted considering a range of limitations. First, interpretation should be assumed as an association and should not imply any causality. Second, most of the information on parental characteristics were self-reported by parents, and mothers responded on behalf of their children; thus, there is a chance of recall errors and/or social desirability bias. Third, some studies have questioned the measurement error of HemoCue device (Karakochuk et al., 2019). Therefore, a cautious interpretation of the reported haemoglobin levels is suggested. Finally, for the measurement of clinical anthropometric biochemical indicators such as height, weight, anaemia, diabetes mellitus and hypertension, all precautions were taken to ensure precision of measurement with stringent monitoring (International Institute for Population Sciences, 2014). However, one cannot exclude the possibility of measurement error. Future studies on this issue should focus on analysing robust cohort data to estimate the effects of parental characteristics on the health of their children.

This study revealed that with father's higher age, children were less likely to experience any anthropometric failure and any anaemia (for age group 30-39 years). This association is consistent with an earlier study, which showed that older fathers were more likely to be involved in their children's healthcare (Moore and Kotelchuck, 2004), thus reducing the odds of morbidity among children. The analysis also revealed that children born to fathers who got married at age $<18$ years had higher likelihood of mortality. Although the adverse effects of low maternal age at marriage on child mortality (Raj et al., 2010) have been studied widely, the adverse effects of a father's low age at marriage on under-five mortality in India remain understudied. To our knowledge, no empirical studies were available to show the detrimental effect of $<18$ years of age at marriage of fathers on offspring health in India (Jejeebhoy, 2019). The role of parental education on child health has been studied globally. Our analysis showed that parental education had a protective association with any anthropometric failure, any anaemia and under-five mortality. Parental education is a proxy indicator for economic opportunities and appropriate health knowledge to take care of their own health and the health of their children (Aslam and Kingdon, 2012). Earlier studies on India and other low- and middle-income countries have shown that paternal education is important for reduction in child undernutrition (Vollmer et al., 2017), and educated parents are more likely to ensure better nutrition for their children (Alderman and Headey, 2017).

Furthermore, our analysis revealed that with increasing maternal height, children had lower odds of any anthropometric failure and were less likely to experience under-five mortality. This finding is consistent with studies conducted in India (Subramanian et al., 2009) and in 54 low- and middle-income countries (Özaltin et al., 2010). Earlier studies on the association between parental BMI and child health (undernutrition) demonstrated that intergenerational associations in nutritional status were not driven by maternal intrauterine influences (Subramanian et al., 2010). Similarly, findings of this study suggest that increased BMI of fathers $(18.50$ through $\geq 30)$ and mothers was associated with lower anthropometric failure, whereas mothers' BMI (18.50 through 29.99) was associated with lower odds of anaemia.

Of behavioural lifestyle indicators, the results indicate that the use of tobacco by mothers was associated with increased underfive mortality. This finding concurs with studies conducted in sub-Saharan African countries (Akinyemi et al., 2016), Southeast Asian Countries (Andriani et al., 2019) and India, where it was suggested that smoking among mothers could lead to adverse pregnancy outcomes (Suliankatchi and Sinha, 2016). Having NCDs such as diabetes among fathers was associated with increased risk of under-five mortality. Although a genetic 
Table 3 Association between parental characteristics and any anaemia (aged 6-59 months).

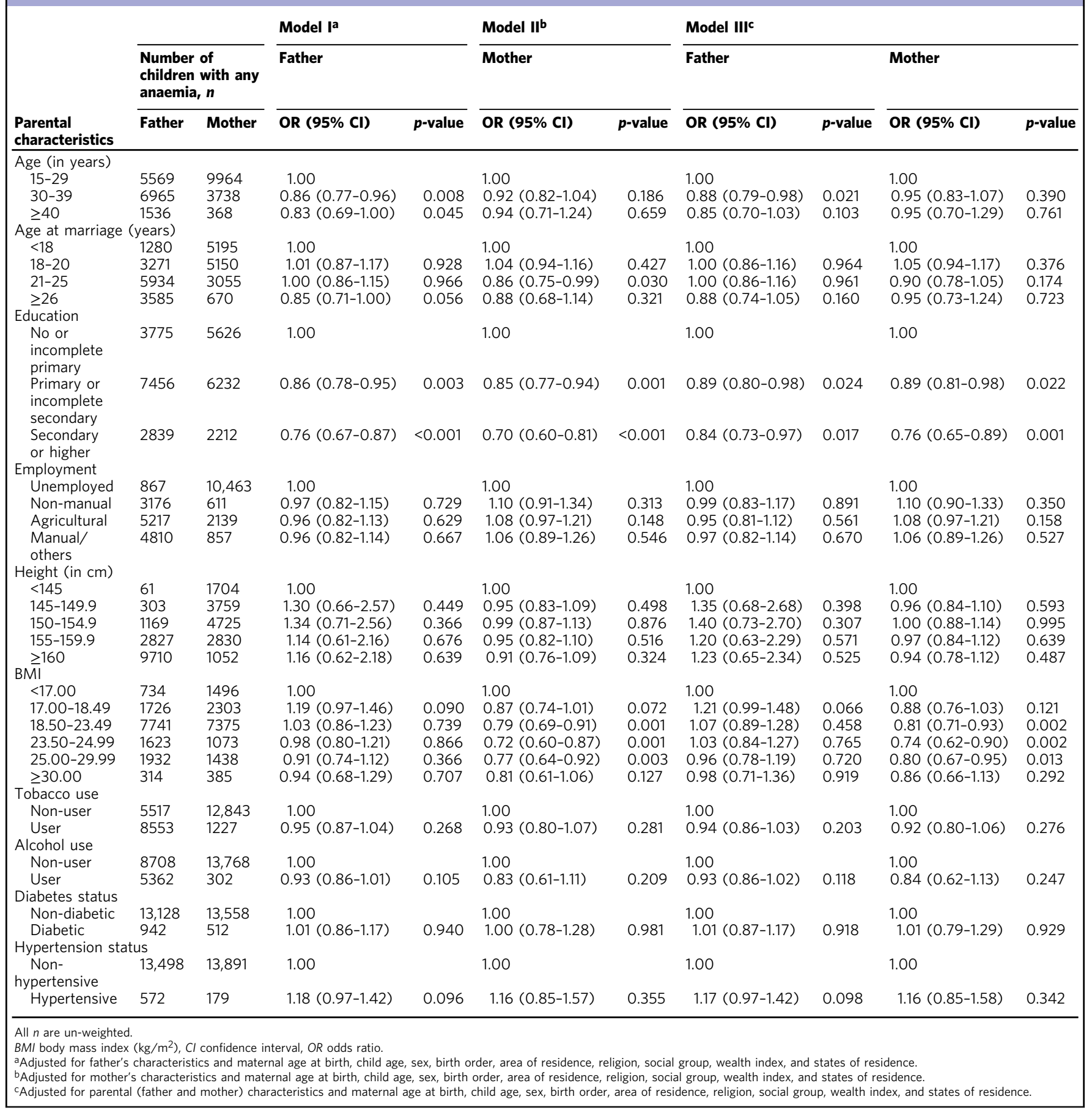

disposition could potentially explain the likelihood of poor health outcomes of children of diabetic fathers (van Esch et al., 2010), this finding needs further exploration on all the possible pathways for which this relationship could be true. In addition, our multivariable analysis showing that children born to hypertensive mothers had lower odds of experiencing any anthropometric failure also requires further investigation, and this finding should be interpreted with caution.

\section{Conclusion}

To conclude, this study highlights that early age at marriage $(<18$ years) among men could be detrimental for the survival of their children. Education of parents was protective for any anthropometric failure, any anaemia and mortality of their children. As maternal height is a protective factor for any anthropometric failure and under-five mortality, this study could be valuable for pre-pregnancy preparation of prospective mothers with relatively shorter height while ensuring that proper nutrition and healthcare is available for children born to mothers with short stature. As use of tobacco by mothers and fathers being diabetic are associated with under-five mortality, a greater focus on mandatory tobacco cessation counselling for women and diabetes control programmes for men could be effective interventions. 


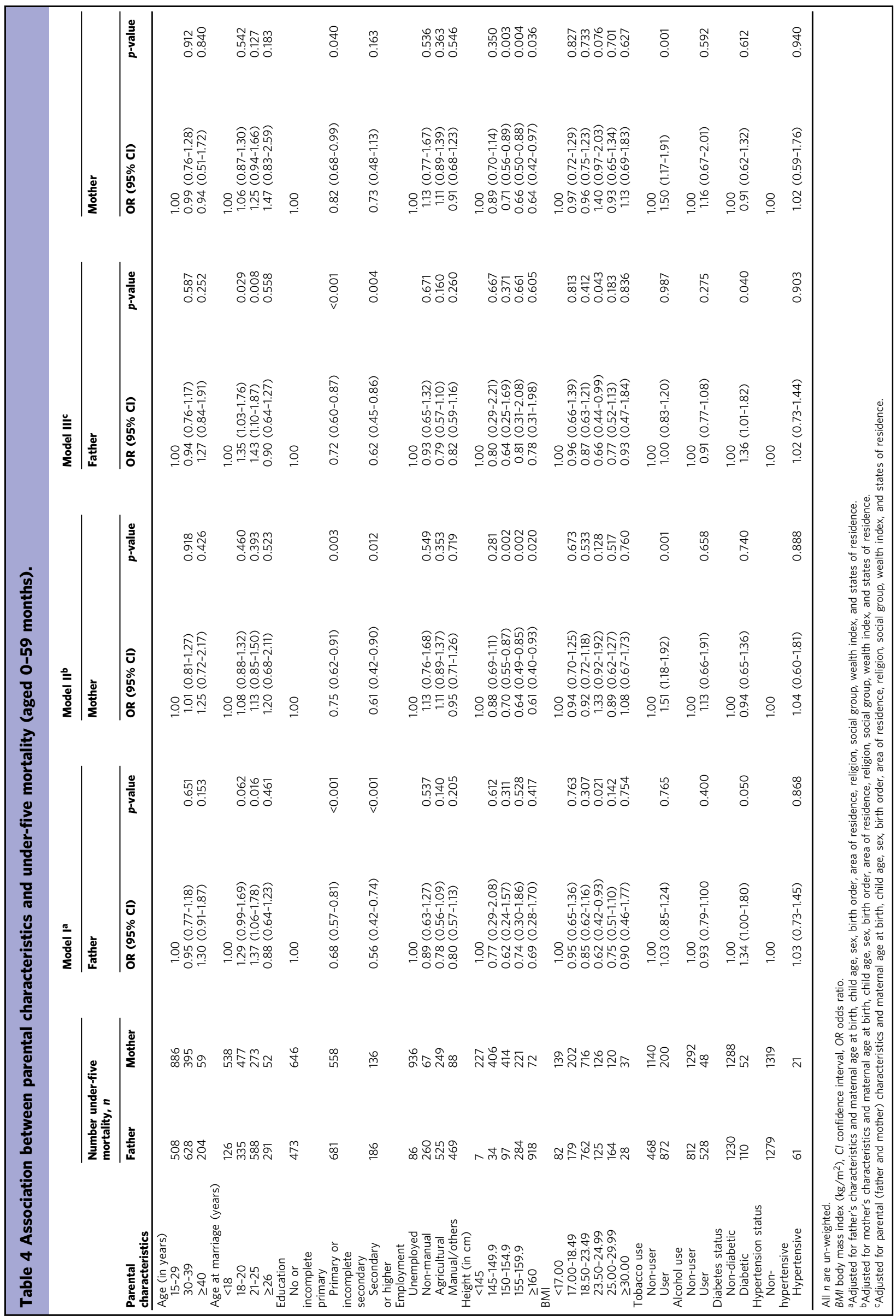




\section{Data availability}

The datasets analysed during the current study are available in The DHS Program repository, https://dhsprogram.com/.

Received: 15 April 2021; Accepted: 12 January 2022;

Published online: 01 February 2022

\section{References}

Akinyemi JO, Adedini SA, Wandera SO et al. (2016) Independent and combined effects of maternal smoking and solid fuel on infant and child mortality in sub-Saharan Africa. Trop Med Int Health 21(12):1572-1582

Alderman H, Headey DD (2017) How important is parental education for child nutrition? World Dev 94:448-464

Andriani H, Putri S, Kosasih RI et al. (2019) Parental smoking and under-five child mortality in Southeast Asia: evidence from Demographic and Health Surveys. Int J Environ Res Public Health 16(23):4756

Aslam M, Kingdon GG (2012) Parental education and child health - understanding the pathways of impact in Pakistan. World Dev 40:2014-2032

Balaj M, York HW, Sripada K et al. (2021) Parental education and inequalities in child mortality: a global systematic review and meta-analysis. Lancet 398(10300):608-620

Bauman LJ, Silver EJ, Stein RE (2006) Cumulative social disadvantage and child health. Pediatrics 117(4):1321-1328

Bhatta DN, Glantz S (2019) Parental tobacco use and child death: analysis of data from demographic and health surveys from South and South East Asian countries. Int J Epidemiol 48(1):199-206

Caleyachetty R, Tait CA, Kengne AP et al. (2014) Tobacco use in pregnant women: analysis of data from Demographic and Health Surveys from 54 low-income and middle-income countries. Lancet Glob Health 2(9):e513-e520

Case A, Lubotsky D, Paxon C (2002) Economic status and health in childhood: the origins of the gradient. Am Econ Rev 92(5):1308-1334

Case A, Paxson C (2002) Parental behavior and chid health. Health Aff (Millwood) 21(2):164-178

Chatterjee S, Hadi AS (2013) Regression analysis by example, 5th edn. Wiley

Chernausek SD, Arslanian S, Caprio S et al. (2016) Relationship between parental diabetes and presentation of metabolic and glycemic function in youth with type 2 diabetes: baseline findings from the TODAY trial. Diabetes Care 39(1):110-117

Corsi DJ, Subramanian SV, Ackerson LK et al. (2015) Is there a greater maternal than paternal influence on offspring adiposity in India? Arch Dis Child 100(10):973-979

Cutler DM, Lleras-Muney A, Vogl T (2008) Socioeconomic status and health: dimensions and mechanisms. National Bureau of Economic Research. Working Paper 14333

Faber T, Kumar A, Mackenbach JP et al. (2017) Effect of tobacco control policies on perinatal and child health: a systematic review and meta-analysis. Lancet Public Health 2(9):e420-e437

Fall CH, Sachdev HS, Osmond C et al. (2015) Association between maternal age at childbirth and child and adult outcomes in the offspring: a prospective study in five low-income and middle-income countries (COHORTS collaboration). Lancet Glob Health 3(7):e366-77

Finlay JE, Özaltin E, Canning D (2011) The association of maternal age with infant mortality, child anthropometric failure, diarrhoea and anaemia for first births: evidence from 55 low- and middle-income countries. BMJ Open 1(2): $\mathrm{e} 000226$

Gastón CM, Misunas C, Cappa C (2019) Child marriage among boys: a global overview of available data. Vulnerable Child Youth Stud 14(3):219-228

Geldsetzer P, Manne-Goehler J, Theilmann M et al. (2018) Diabetes and hypertension in India: a nationally representative study of 1.3 million adults. JAMA Intern Med 178(3):363-372

Gupta A, Cleland J, Sekher TV (2021) Effects of parental stature on child stunting in India. J Biosoc Sci. https://doi.org/10.1017/S0021932021000304

Huq T, Alexander EC, Manikam L et al. (2021) A systematic review of household and family alcohol use and childhood neurodevelopmental outcomes in lowand middle-income countries. Child Psychiatry Hum Dev 52(6):1194-1217

ICF (2018) Demographic and Health Surveys Standard Recode Manual for DHS 7. The Demographic and Health Surveys Program. ICF, Rockville, Maryland, USA

International Institute for Population Sciences (2014) National Family Health Survey (NFHS-4), 2015-16: India, Clinical Anthropometric Biochemical (CAB) Manual. International Institute for Population Sciences, Mumbai

International Institute for Population Sciences and ICF (2017) National Family Health Survey (NFHS-4), 2015-16: India. International Institute for Population Sciences, Mumbai
Jejeebhoy SJ (2019) Ending Child Marriage in India, Drivers and Strategies. UNICEF, New Delhi

Jose JP, Cherayi SJ (2020) Effect of parental alcohol abuse severity and child abuse and neglect on child behavioural disorders in Kerala. Child Abuse Negl 107:104608

Karakochuk CD, Hess SY, Moorthy D et al. (2019) Measurement and interpretation of hemoglobin concentration in clinical and field settings: a narrative review. Ann N Y Acad Sci 1450(1):126-146

Kumar P, Chauhan S, Patel R et al. (2021a) Anaemia among mother-father-child pairs in India: examining co-existence of triple burden of anaemia in a family. BMC Public Health 21(1):1341

Kumar P, Chauhan S, Patel R et al. (2021b) Prevalence and factors associated with triple burden of malnutrition among mother-child pairs in India: a study based on National Family Health Survey 2015-16. BMC Public Health 21(1):391

Landberg J, Danielsson AK, Falkstedt D et al. (2018) Fathers' alcohol consumption and long-term risk for mortality in offspring. Alcohol Alcohol 53(6):753-759

Leonardi-Bee J, Britton J, Venn A (2011) Secondhand smoke and adverse fetal outcomes in nonsmoking pregnant women: a meta-analysis. Pediatrics 127(4):734-741

Miliku K, Bergen NE, Bakker H et al. (2016) Associations of maternal and paternal blood pressure patterns and hypertensive disorders during pregnancy with childhood blood pressure. J Am Heart Assoc 5(10):e003884

Miranda JO, Cerqueira RJ, Barros $\mathrm{H}$ et al. (2019) Maternal diabetes mellitus as a risk factor for high blood pressure in late childhood. Hypertension 73(1):e1-e7

Moore T, Kotelchuck M (2004) Predictors of urban fathers' involvement in their child's health care. Pediatrics 113(3 Pt 1):574-580

Nicoletti D, Appel LD, Siedersberger Neto P et al. (2014) Maternal smoking during pregnancy and birth defects in children: a systematic review with metaanalysis. Cad Saude Publica 30:2491-2529

Nykjaer C, Alwan NA, Greenwood DC et al. (2014) Maternal alcohol intake prior to and during pregnancy and risk of adverse birth outcomes: evidence from a British cohort. J Epidemiol Community Health 68(6):542-549

Özaltin E, Hill K, Subramanian SV (2010) Association of maternal stature with offspring mortality, underweight, and stunting in low- to middle-income countries. JAMA 303(15):1507-1516

Patel R, Srivastava S, Kumar P et al. (2020) Factors associated with double burden of malnutrition among mother-child pairs in India: A study based on National Family Health Survey 2015-16. Child Youth Serv Rev 116:105256

Perez-Alvarez P, Favara M (2019) Maternal age and offspring human capital in India. IZA Institute of Labour Economics, Bonn, Germany

Puri P, Khan J, Shil A et al. (2020) A cross-sectional study on selected child health outcomes in India: Quantifying the spatial variations and identification of the parental risk factors. Sci Rep 10(1):6645

Rai RK, Kumar SS, Parasannanavar DJ et al. (2022) Tipping the scale: the role of a national nutritional supplementation programme for pregnant mothers in reducing low birth weight and neonatal mortality in India. Br J Nutr 127(2):289-297

Rai RK, Singh L, Singh PK (2017) Is maternal body mass index associated with neonatal mortality? A pooled analysis of nationally representative data from nine Asian countries. Nutrition 41:68-72

Raj A, Saggurti N, Winter M et al. (2010) The effect of maternal child marriage on morbidity and mortality of children under 5 in India: cross sectional study of a nationally representative sample. BMJ 340:b4258

Semba RD, Kalm LM, de Pee S et al. (2007) Paternal smoking is associated with increased risk of child malnutrition among poor urban families in Indonesia. Public Health Nutr 10(1):7-15

Shajan J, Sumalatha BS (2020) Maternal employment and children's health in India: An exploratory analysis. J Public Aff. https://doi.org/10.1002/pa.2580

Sinha S, Aggarwal AR, Osmond C et al. (2016) Maternal age at childbirth and perinatal and under five mortality in a prospective birth cohort from Delhi. Indian Pediatr 53(10):871-877

Srivastava S, Upadhyay AK (2022) A success story of reduction in childhood stunting and underweight in India: analysis of pooled data from three rounds of Indian Demographic and Health Surveys (1998-2016). J Biosoc Sci 54(1):106-123

StataCorp (2015) Stata Statistical Software: Release 14. Stata Corp LP, College Station, TX

Subramanian SV, Ackerson LK, Davey Smith G et al. (2009) Association of maternal height with child mortality, anthropometric failure, and anemia in India. JAMA 301(16):1691-1701

Subramanian SV, Ackerson LK, Smith GD (2010) Parental BMI and childhood undernutrition in India: an assessment of intrauterine influence. Pediatrics 126(3):e663-e671

Suliankatchi RA, Sinha DN (2016) The human cost of tobacco chewing among pregnant women in india: a systematic review and meta-analysis. J Obstet Gynaecol India c66(Suppl 1):161-166 
Sullivan KM, Mei Z, Grummer-Strawn L et al. (2008) Haemoglobin adjustments to define anaemia. Trop Med Int Health 13(10):1267-1271

Uehara Y, Shin WS, Watanabe T et al. (1998) A hypertensive father, but not hypertensive mother, determines blood pressure in normotensive male offspring through body mass index. J Hum Hypertens 12(7):441-445

van Esch SC, Cornel MC, Snoek FJ (2010) "I am pregnant and my husband has diabetes. Is there a risk for my child?" A qualitative study of questions asked by email about the role of genetic susceptibility to diabetes. BMC Public Health 10:688

Vollmer S, Bommer C, Krishna A et al. (2017) The association of parental education with childhood undernutrition in low- and middle-income countries: comparing the role of paternal and maternal education. Int J Epidemiol 46(1):312-323

Vyas S, Kumaranayake L (2006) Constructing socio-economic status indices: how to use principal components analysis. Health Policy Plan 21(6):459-468

Wells JCK, Nesse RM, Sear R et al. (2017) Evolutionary public health: introducing the concept. Lancet 390(10093):500-509

WHO Multicentre Growth Reference Study Group (2006) WHO child growth standards: length/ height-for-age, weight for-age, weight-for-length and body mass index for age: methods and development. World Health Organization, Geneva

World Health Organization (2011) Haemoglobin concentrations for the diagnosis of anaemia and assessment of severity. Vitamin and Mineral Nutrition Information System. World Health Organization (WHO/NMH/NHD/MNM/ 11.1), Geneva

World Health Organization (2021) Tobacco control to improve child health and development: thematic brief. World Health Organization, Geneva

Zhuge Y, Qian H, Zheng X et al. (2020) Effects of parental smoking and indoor tobacco smoke exposure on respiratory outcomes in children. Sci Rep 10(1):4311

\section{Acknowledgements}

RKR received a research grant from the Department of Health and Family Welfare, Government of West Bengal, India [memo number-114-P\&B/ HFW-27011/114/2019NHM SEC]. The article processing charges was borne by open access funds of the University of Goettingen, Germany.

\section{Author contributions}

RKR, SVS and SV conceived the study and devised the analytical approach. RKR performed the analysis and prepared the first draft. SVS and SV critically reviewed findings and revised the manuscript. All authors approved the final manuscript.

\section{Funding}

Open Access funding enabled and organized by Projekt DEAL.

\section{Competing interests}

The authors declare no competing interests.

\section{Ethical approval}

Prior to conducting the National Family Health Survey, ethical approval was obtained by the International Institute for Population Sciences from an independent ethics review committee constituted by the Ministry of Health and Family Welfare, Government of India. This work complies with the ethical standards of the relevant national and institutional committees on human experimentation and with the Helsinki Declaration of 1975 , as revised in 2008. At the official website of the DHS programme-https:// dhsprogram.com/, NFHS-4 datasets are available to the public for use by researchers with all participant identifiers removed, thus this study was exempted from an additional ethics committee review.

\section{Informed consent}

Written and/or informed consent was obtained from all study participants and/or their legal guardians.

\section{Additional information}

Supplementary information The online version contains supplementary material available at https://doi.org/10.1057/s41599-022-01054-2.

Correspondence and requests for materials should be addressed to Rajesh Kumar Rai.

Reprints and permission information is available at http://www.nature.com/reprints

Publisher's note Springer Nature remains neutral with regard to jurisdictional claims in published maps and institutional affiliations.

Open Access This article is licensed under a Creative Commons Attribution 4.0 International License, which permits use, sharing, adaptation, distribution and reproduction in any medium or format, as long as you give appropriate credit to the original author(s) and the source, provide a link to the Creative Commons license, and indicate if changes were made. The images or other third party material in this article are included in the article's Creative Commons license, unless indicated otherwise in a credit line to the material. If material is not included in the article's Creative Commons license and your intended use is not permitted by statutory regulation or exceeds the permitted use, you will need to obtain permission directly from the copyright holder. To view a copy of this license, visit http://creativecommons.org/ licenses/by/4.0/.

(c) The Author(s) 2022 